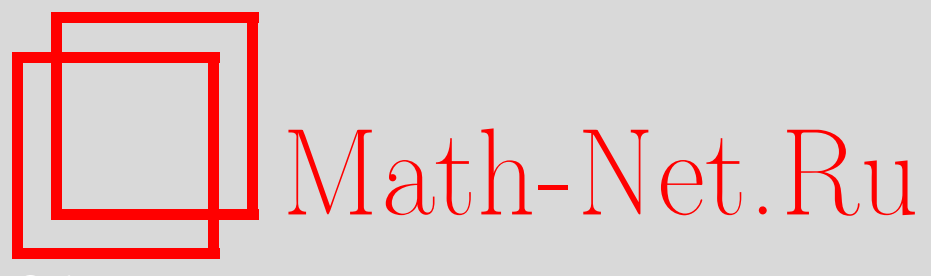

И. Н. Бройтигам, К. А. Мирзоев, Т. А. Сафонова, Аналог теоремы Орлова об индексе дефекта для матричных дифференциальных операторов второго порядка, Матем. заметки, 2015, том 97, выпуск 2, 314-317

DOI: https://doi.org/10.4213/mzm10578

Использование Общероссийского математического портала Math-Net.Ru подразумевает, что вы прочитали и согласны с пользовательским соглашением http://www . mathnet.ru/rus/agreement

Параметры загрузки:

IP: 34.239 .49 .27

26 апреля 2023 г., 12:11:44

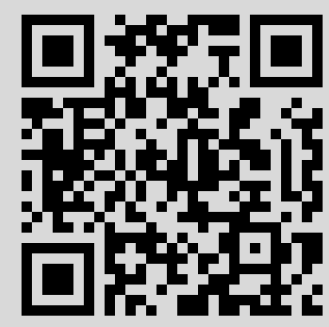




\section{Аналог теоремы Орлова об индексе дефекта для матричных дифференциальных операторов второго порядка}

\section{И. Н. Бройтигам, К. А. Мирзоев, Т. А. Сафонова}

1. Введение. В работе Орлова [1] был найден класс линейных дифференциальных операторов с вещественными аналитическими коэффициентами, дефектные числа которых определяются как число корней некоторого явно выписываемого полинома, лежащих в левой полуплоскости, а в работе [2] показано, что результат Орлова остается справедливым и для некоторых операторов с неаналитическими коэффициентами. Далее, в работе [3], в частности, были существенно расширены классы операторов, для которых справедливы утверждения теоремы Орлова.

Цель данной заметки - установить аналог теоремы Орлова для операторов, порожденных матричными линейными квазидифференциальными выражениями второго порядка.

2. Матричные квазидифференциальные выражения второго порядка. Пусть $P(x), Q(x)$ и $R(x)$ - матричные функции порядка $n, n \in \mathbb{N}$, определенные на множестве $I:=[1 ;+\infty)$, причем $P(x)$ - невырожденная матрица и $P(x)$ и $Q(x)$ - эрмитовы матрицы.

Условие (А). Предположим, что комплекснозначные функиии $p_{i j}(x), q_{i j}(x) u r_{i j}(x)$, $i, j=1,2, \ldots, n,-$ элементы матрии $P^{-1}(x), Q(x)$ и $R(x)$ соответственно - определены, измеримы на множестве I и суммируемы на каждом его замкнутом конечном интервале $\left(p_{i j}(x), q_{i j}(x), r_{i j}(x) \in L_{\mathrm{loc}}^{1}(I)\right)$.

Определим первую квазипроизводную заданной на $I$ вектор-функции $y(x)=\left(y_{1}(x)\right.$, $\left.y_{2}(x), \ldots, y_{n}(x)\right)^{t}$ с локально абсолютно непрерывными координатами $\left(y_{i}(x) \in A C_{\mathrm{loc}}(I)\right)$, где $t$ - символ транспонирования, посредством матриц $P$ и $R$, полагая

$$
y^{[1]}:=P\left(y^{\prime}-R y\right) .
$$

Далее, считая, что вектор-функция $y^{[1]}=\left(y_{1}^{[1]}, y_{2}^{[1]}, \ldots, y_{n}^{[1]}\right)^{t}$ уже определена, $y_{i}^{[1]} \in A C_{\text {loc }}(I)$, определим вторую квазипроизводную $y^{[2]}$ вектор-функции $y$ и квазидифференциальное выражение $l[y]$, полагая соответственно

$$
y^{[2]}:=\left(y^{[1]}\right)^{\prime}+R^{*} y^{[1]}-Q y,
$$

где ${ }^{*}$ - символ сопряжения и

$$
l[y](x):=-y^{[2]}(x), \quad x \in I .
$$

Множество вектор-функций

$$
\mathscr{D}:=\left\{y(x) \mid y_{i}(x), y_{i}^{[1]}(x) \in A C_{\mathrm{loc}}(I), i=1,2, \ldots, n\right\},
$$

очевидно, является областью определения выражения $l[y](x)$, а из условия (А) следует, что для любой функции $y(x) \in D$ выражение $l[y](x)$ существует п.в. на $I$ и координаты $l[y]$

Работа первого автора выполнена при поддержке Министерства образования и науки РФ и Германской службы академических обменов (DAAD) (программа Михаил Ломоносов, 325-A/13/ 74976). Работа второго автора выполнена при поддержке Российского научного фонда (грант № 14-11-00754). Работа третьего автора выполнена при поддержке Российского фонда фундаментальных исследований (гранты №№ 14-01-31136-мол_а и 14-01-00349).

DOI: $10.4213 / \mathrm{mzm} 10578$ 
локально интегрируемы. Кроме того, для любых двух вектор-функций $f, g \in \mathscr{D}$ справедлив векторный аналог тождества Грина

$$
\int_{\alpha}^{\beta}\{(l[f], g)-(f, l[g])\}=[f, g](\beta)-[f, g](\alpha), \quad 1 \leqslant \alpha \leqslant \beta<\infty,
$$

где $(g, h)=\sum_{s=1}^{n} g_{s} \bar{h}_{s}-$ скалярное произведение векторов $g$ и $h$, а форма $[f, g]$ определятся равенством $[f, g](x):=\left(f^{[1]}, g\right)-\left(f, g^{[1]}\right)$.

Рассмотрим матричное квазидифференциальное уравнение

$$
l[y]=\lambda y,
$$

где $\lambda \in C$.

Отметим, что это уравнение равносильно системе дифференциальных уравнений 1-го порядка

$$
\mathbf{y}^{\prime}=(F-\Lambda) \mathbf{y}
$$

где

$$
F=\left(\begin{array}{cc}
R & P^{-1} \\
Q & -R^{*}
\end{array}\right), \quad \Lambda=\left(\begin{array}{cc}
O & O \\
\lambda I_{n} & O
\end{array}\right), \quad \mathbf{y}=\left(y_{1}, \ldots, y_{n}, y_{1}^{[1]}, \ldots, y_{n}^{[1]}\right)^{t},
$$

а символами $O$ и $I_{n}$ обозначены нулевая и единичная матрицы порядка $n$ соответственно. Равносильность уравнений (3) и (4) понимается в том смысле, что если $y(x)$ является векторным решением системы (3), то $2 n$-компонентный вектор-столбец у является решением (4) и наоборот, если $2 n$-компонентный вектор-столбец $\mathbf{y}-$ решение системы (4), то первые его $n$ компонент - решение уравнения (3).

Отметим также, что условие (А) обеспечивает справедливость теоремы существования и единственности решения задачи Коши для системы (4), поставленной в любой точке интервала $I$.

3. Минимальный оператор $L_{0}$. Индекс дефекта. Пусть $L_{n}^{2}(I)$ - гильбертово пространство $n$-компонентных комплекснозначных вектор-функций, измеримых по Лебегу на $I$, у которых сумма квадратов модулей координат интегрируема по Лебегу на $I$. С помощью таких же рассуждений, как и в [4], с использованием формулы (2), определяется минимальный замкнутый симметрический оператор $L_{0}$, порожденный выражением $l[y]$ в пространстве $L_{n}^{2}(I)$. Согласно [4]-[6] дефектные числа $n_{+}$и $n_{-}$оператора $L_{0}$ совпадают с максимальным числом линейно независимых решений уравнения (3), когда параметр $\lambda$ берется из верхней $(\operatorname{Im} \lambda>0)$ или нижней $(\operatorname{Im} \lambda<0)$ полуплоскости соответственно, удовлетворяют неравенствам $n \leqslant n_{+}, n_{-} \leqslant 2 n$ и, кроме того, $n_{+}=2 n$ тогда и только тогда, когда $n_{-}=$ $2 n$. Пара чисел $\left(n_{+}, n_{-}\right)$называется индексом дефекта оператора $L_{0}$.

\section{4. Аналог теоремы Орлова об индексе дефекта оператора $L_{0}$.}

УСловие (В). Предположим, что при всех $x \geqslant 1$ и некотором $\nu>0$ матричные функици $P^{-1}(x), Q(x)$ и $R(x)$ представимы в виде

$$
P^{-1}(x)=x^{-\nu-2}\left(P_{0}+P_{1}(x)\right), \quad Q(x)=x^{\nu}\left(Q_{0}+Q_{1}(x)\right), \quad R(x)=x^{-1}\left(R_{0}+R_{1}(x)\right),
$$

где $P_{0}, Q_{0}$ и $R_{0}$ - квадратные числовые матрицы порлдка $n$, а $P_{1}(x), Q_{1}(x)$ и $R_{1}(x)$ матричные функиии того же порядка.

Обозначим через $D$ блочно-диагональную матрицу вида

$$
D=\left(\begin{array}{cc}
x^{-1 / 2} I_{n} & O \\
O & x^{\nu+1 / 2} I_{n}
\end{array}\right)
$$

и в системе (4) сделаем замену $\mathbf{y}=D Y$. Простые рассуждения показывают, что неизвестная вектор-функция $Y$ удовлетворяет системе дифференциальных уравнений

$$
Y^{\prime}=x^{-1}(A+B(x)) Y,
$$


где $A$ - квадратная числовая матрица $2 n$-го порядка и $B(x)$ - матричная функция того же порядка, которые в блочном представлении имеют вид

$$
A=\left(\begin{array}{cc}
R_{0}+\frac{1}{2} I_{n} & P_{0} \\
Q_{0} & -R_{0}^{*}-\left(\nu+\frac{1}{2}\right) I_{n}
\end{array}\right), \quad B=\left(\begin{array}{cc}
R_{1}(x) & P_{1}(x) \\
Q_{1}(x)-\frac{\lambda}{x^{\nu}} I_{n} & -R_{1}^{*}(x)
\end{array}\right) .
$$

Предположим далее, что матричные функции $P_{1}(x), Q_{1}(x)$ и $R_{1}(x)$ таковы, что выполняется

УСловие (С). Интеграль

$$
\int_{1}^{+\infty} \frac{\ln ^{r}(x)}{x}\left|P_{1}(x)\right| d x, \quad \int_{1}^{+\infty} \frac{\ln ^{r}(x)}{x}\left|Q_{1}(x)\right| d x, \quad \int_{1}^{+\infty} \frac{\ln ^{r}(x)}{x}\left|R_{1}(x)\right| d x
$$

сходятся, где $r+1$ - максимальная кратность характеристического корня матрицы $A$, $a|\cdot|$ - норма матрицы, определяемая как сумма модулей всех ее элементов.

Условие (С) позволяет, применив результат задачи из [7] (см. задача 35, гл. III, с. 120), получить главный член асимптотики некоторой фундаментальной матрицы системы (5) nри $x \rightarrow+\infty$. Из этого уже легко определить и главный член асимптотики некоторой фундаментальной матрицы уравнения (3).

Эти асимптотические формулы позволяют сформулировать и доказать аналог теоремы Орлова для определения дефектных чисел оператора $L_{0}$, порожденного матричным квазидифференциальным выражением (3), т.е. справедлива следующая

Теорема 1. Пусть выполнены условия $(A)-(C)$, тогда дефектные числа $n_{+} u n_{-}$оператора $L_{0}$ равны между собой и определяются как число корней многочлена $\mathscr{F}(z, \nu):=$ $\operatorname{det}\left(A-z I_{2 n}\right)$, лежащих в области $\operatorname{Re} z<0$.

5. Пример. Пусть $n=2$ и $R(x)=O$. Тогда квазидифференциальное выражение (1) и многочлен $\mathscr{F}(z, \nu)$ запишутся в виде

$$
\begin{gathered}
l[y](x)=-\left(P y^{\prime}\right)^{\prime}+Q y, \quad x \in I \\
\mathscr{F}(z, \nu)=\left[\left(z+\frac{\nu}{2}\right)^{2}-\left(\frac{\nu+1}{2}\right)^{2}\right]^{2} \\
\quad-\left[\left(z+\frac{\nu}{2}\right)^{2}-\left(\frac{\nu+1}{2}\right)^{2}\right] \times \operatorname{sp}\left(P_{0} \cdot Q_{0}\right)+\operatorname{det} P_{0} \times \operatorname{det} Q_{0} .
\end{gathered}
$$

Отметим, что многочлен $\mathscr{F}(z, \nu)$ является произвольным квадратным трехчленом относительно $(z+\nu / 2)^{2}$, поэтому число корней этого многочлена, лежащих в левой полуплоскости, за счет выбора элементов матриц $P_{0}$ и $Q_{0}$ может быть сделано любым из чисел 2,3 и 4 .

ЗАмечание. Выбирая матрицы $P, Q$ и $R$ надлежащим образом, можно доказать, что аналог теоремы 1 остается в силе и для оператора $L_{0}$, порожденного выражением вида

$$
l[y](x)=-\left(P y^{\prime}\right)^{\prime}+Q^{\prime} y, \quad x \in I,
$$

где всюду производные понимаются в обобщенном смысле. 


\section{СПИСОК ЦИТИРОВАННОЙ ЛИТЕРАТУРЫ}

[1] С. А. Орлов, Докл. АН СССР, 92:3 (1953), 483-486. [2] Ф. А. Неймарк, "Об индексе дефекта дифференциального оператора", УМН, 17:4 (1962), 157-163. [3] К. А. Мирзоев, Докл. РАН, 380:5 (2001), 591-595. [4] М. А. Наймарк, Линейнъе дифференииалъные операторы, Наука, М., 1969. [5] В. П. Серебряков, Изв. вузов. Матем., 2000, № 3, 48-53. [6] R. L. Anderson, Canad. J. Math, 28:5 (1976), 905-914. [7] Э. А. Коддингтон, Н. Левинсон, Теория обыкновенных дифференииальных уравнений, ИЛ, М., 1958.

И.Н. Бройтигам

Северный (Арктический) федеральный

университет им. М. В. Ломоносова, г. Архангельск

E-mail: irinadolgih@rambler.ru

\section{К. А. Мирзоев}

Московский государственный

университет им. М. В. Ломоносова

E-mail: mirzoev.karahan@mail.ru

\section{Т. А. Сафонова}

Северный (Арктический) федеральный

университет им. М. В.Ломоносова, г. Архангельск

E-mail: tanya.strelkova@rambler.ru 